\title{
Smart Sandal with IoT for Women's Safety and Empowerment
}

\author{
Mir Sajjad Hussain Talpur ${ }^{1}$, Raheel Sarwar ${ }^{1}$, Abida Luhrani ${ }^{1}$, Samina Rajper ${ }^{3}$, Fauzia Talpur ${ }^{2}$, Hina \\ Rehman $^{1}$, Junaid Chandio ${ }^{1}$, Shakir Hussain Talpur ${ }^{1}$, Erum Saba Chang ${ }^{1}$, Aijaz Ahmed Kalhoro ${ }^{3}$ \\ ${ }^{1}$ Information Technology Centre, Sindh Agriculture University, TandoJam, mirsajjadhussainsau.edu.pk \\ ${ }^{2}$ Sindh University Laar Campus Badin, Pakistan, fozia.talpur@usindh.edu.pk \\ ${ }^{3}$ Computer Science Department, Shah Abdul Latif University, Pakistan
}

\section{ABSTRACT}

The modern world with giant bustling cities is a world in which everyone is busy with their work; nobody knows what is happening in society. In such a busy environment, several hooligans try to harass women by various unfair means. Women, as we all know, are working in the entire arena of modern tech-related fields. Women workday and night to support their livelihood. These women are vulnerable to attacks and assaults, and security and safety are major concerns for them. Women's rights are neglected in the patriarchal society of Pakistan; hence the crimes against women are rising to alarming levels. Violence against women is widespread and pervasive not only in Pakistan's cities and villages, but throughout the world. Official statistics are always manipulated by the governmental departments to hide their incompetence and inadequacies. According to a recent survey, it found that 84 percent of the women who experienced harassment in workplaces were an old group of 25 to 35 years, who are mostly full-time workers and students. The safety concerns for these women are rising due to the significant cases of harassment and rapes. IoT is a relatively new field in information technology which is related to computing accessories and devices which can also be called digital machines and also several other objects like a wristwatch, glasses, smart sandals, soil moisture sensors, etc. Through IoT, we can manufacture our desired devices with the help of sensors, circuits, and monitoring and evaluation is made very convenient. The concept of the Internet of things is a demand-driven concept which will change the whole use of information technology. This proposed system is named the Smart sandal. The primary purpose of this smart sandal is to provide women with a gadget for their security. It is very easy to wear, and it will help in the family. Immediately the button is pushed, the shock will be felt, and notification will be sent by using GSM on the specific number stored, and the location of those women is traced with the help of Global Positioning System (GPS).
Key words : Internet of things, Smart sandal, Women safety, Sensors.

\section{INTRODUCTION}

The population explosion in the modern era is alarming; the current population is around 7 billion people, with a projected increase to 9 billion people in the next 30 years. Many women face severe discrimination at different stages of their lives. Women make a significant contribution to our dwindling economy. Women used to sit at home and perform domestic duties, but times have changed, and women now play a larger role in our society. As a result, women's contribution to Pakistan's economic development is critical. Women's rights are largely ignored and violated on a massive scale, and they must be protected. According to a recent survey, 84 percent of women who have experienced workplace harassment are between the ages of 25 and 35, and are mostly full-time workers and students. Because of the numerous cases of harassment and rape, these women's safety concerns are growing. The 'Internet of Things' is a relatively new term that is gaining traction around the world, including in Pakistan. Advances in IoT devices will help to improve living standards, as well as monitoring and evaluation processes for Pakistan's key stakeholders. The rapid advancement of the Internet of Things in specific areas such as the design of smart, reliable, and low-cost sensors, among other things, will help meet the growing demands of the modern world and related fields. WIFI and BLE, which are required to embed smart sandals, are examples of IoT applications in a wide range of IT fields.

Women's empowerment is a broad term that can refer to a variety of things, including providing women with high-quality education, modern health care, and the ability to obtain high-paying jobs in modern hi-tech institutions. Women's empowerment can also take the form of granting them the right to vote. This fundamental right is denied to a large number of Pakistani women. Not only should they be given this right, but they should also make the voting process 
easier for women. The International Monetary Fund studied women's rights around the world and came to the conclusion that women play a critical role in a country's economic growth, and that women's empowerment would boost GDP by up to $30 \%$. In government employment, women face sexism, and the private sector is reluctant to recruit them until international human rights organizations exert enough leverage. As specified in Article 25 (2) of the constitution, it is the duty of the state to provide equal opportunities to all sectors of society, including women. Regrettably, despite having clear and specific articles in the constitution, women are deprived and discriminated against in various areas of society due to governmental institutions' lack of interest. Women's rights activists and their allies should not only raise awareness about the issue, but also hold rallies, conferences, and debates on empowerment issues on a regular basis. As soon as we take the steps outlined above with zeal and excitement, people will progressively become more conscious of women's rights, and the social attitude around women's rights will change in favor of women's rights.It was Islam who gave full rights to women. Women were even buried alive before pre-Islamic civilization, and the Prophet (P.B.U.H) expressed extreme anger and displeasure in such cases.

On the streets, parks, and public transportation, as well as in and around workplaces and schools, women face physical harassment. It has been discovered that the majority of these cases involve single women. As a result, it is critical to create systems that assist women in fighting even when they are alone. If a system could notify the victim's family, as well as the people around her, about her current location, the victim's chances of being helped would greatly improve. A device like this allows the victim to warn other people within a certain distance, including family members. It's past time for women to make a difference. Women are now involved in every aspect of modern society, from major construction projects to space flights, and from health to natural farming. Women are demonstrating their abilities in any area they join. It's also painful to mention that many women around the world work extra hours in the workplace but are not compensated by big corporations or companies. Even if the companies provide transportation, women should be granted leniency during pregnancy timings and should be provided with complete health care and financial support to the maximum extent possible. We've seen a lot of similar events in recent years where women's safety isn't the only solution to the issue. Women should be given a portable safety device that guarantees their safety. Our project focuses on creating a mobile device focused on IoT solutions that not only assists women in avoiding risky circumstances, but also ensures that women obtain justice. The Internet of Things is a relatively new concept of technology that is gaining traction as time passes. The advent of this tech-related area is critical for women's safety because IoT technology is extremely precise and smart, and it can be carried around without causing problems. When it is worn on the body, the shock is produced when the push button is pressed, and the message is sent via GSM. Women have spread to every corner of the globe as a result of the new technological revolution; this has generated distrust among women about safety concerns, as the supposed harassers are none other than their coworkers. Since women's rights have been subjugated and she is even forced to remain silent on such issues, it is now the duty of every person to protect them. We should make a promise to continue our efforts and make regular innovations for the protection of our women until and until they are protected, because women are a convenient and easy target for criminals. As a result, a minute ago you would have had to lose the button and your surroundings in the spirit of the inventory phone announcement and in the minute of assistance to be relaxed or request that the measurements be taken here. We have a second GPS component on hand to monitor women's movements.

\section{REVIEW OF LITRACTURE}

We talked about the background realities of women's safety and security in this section. Vemu Institute of Technology's G.Bharathi and L. Ramurthy[1] suggested a method for monitoring children. It was a very basic system that could only provide the user's location via GSM. A research paper on an IoT-enabled gadget for children and women was published in 2016. Another IoT-based safety device, Geetha Pratyusha Miriyala [2], was proposed with the help of GPS. The victim's exact location is likely to be discovered, and alerts will be sent to the contacts for assistance as soon as possible. J. Muhammad, Y. Sarosh, M. Muteeb, S. Shahid, and M. Muteeb [3] The Mehfooz Aurat proposes that safe routes be shown throughout Lahore, Pakistan. Another application, SafetiPin Al-Suwaidi and Zemerl [4] et al. et al., was developed by Al-Suwaidi and Zemerl) proposed that the application has solved many problems by utilizing the global positioning system and utilizing the mobile tracking system to locate friends and family. The client-server architecture approach was used in this system. The server allows the client's mobile to register and $\log$ in, and it saves the client's password and credentials in the server's database. The client usually sends the server the location coordinates for tracking. As a result, the server has kept an eye on the area. The application aided in the location of friends and family members.

Chandra et al., Chandra et al., Chandra et al. [5] Proposed a special SMS service solution that has been introduced for Java-based mobile phones that support GPS. The clients in this application share their current locations via SMS on an Internet-based server. On the map, the region has been tracked, allowing the client to provide live streaming. [6] 
Almomani et al., Almomani et al., Almomani et al. He proposes a paper in which he discusses a well-known car management and monitoring device that incorporates an ubiquitous GPS global positioning system. The framework includes a mobile and client-server application that uses GPS on the server side and SMS to manage the user database and information. In the Client Box, there is a GSM modem as well as tracking systems with GPS. All of the necessary information is saved in the server's Internet-based portal, and the credentials are saved in the server's databases. This program was created for the purpose of tracking the driving actions of employees. et. al., M. Navya [7] .et. al., M. Navya [7] .et Proposes a GSM-based GPS system for tracking children's locations. The student's location was indicated by the GPS. The Global System for Mobile Technology is used to communicate student information to their parents. The monitoring system was installed by the school in the control rooms. HuoshengHu [8] Huiyu Zhou A microcontroller chip has been put in the bag with the RF-id reader to schedule the matched books for a day. As a result, the RF-id will determine how many books are missing or not in the pack, as well as whether or not any extra books are present. A beep has signaled to the consumer that an additional book or missing book is not needed for the particular day for the identification alarm. Shibata, Kun [9] A panic button is one of the bag's distinguishing features. In an emergency, such as a kidnapping or robbery, the user should push the button. When the button is pressed, a message is sent to the user's home and police station via Bluetooth and GPS, which automatically detect the user's location. According to the study by Shafaat [10], new technologies have been implemented in the Emirates Transport in Dubai, which will improve traffic safety and improve the transportation service for schoolchildren. It also allows parents to track their children's school bus routes and to map out the position of the school buses. The golden rule, device tracking system, and smart card are the three distinct strategies that make up the tracking system. When a student enters the bus, he or she checks the device's Id, and the same protocol is followed when the student exits the bus.Each parent receives a message when their child enters and exits the bus by scanning their cards. The tracking system has enabled the direction of the bus route as the students enter the bus after scanning a card, and each parent can control the route on their mobile by following the spot. As a result, the other rule, known as the golden rule, has included the golden box located in the bus parking area inside the larger red box, which has cautioned the students against getting around. To save students from the exposure warning, it is necessary to warn them when crossing roads or when the bus is moving in front of them. During the implementation, it was discovered that there was a significant reduction in the number of injuries, with a percentage of 63 , as well as a reduction in the use of private vehicles, which students typically use out of fear of accidents, by about $9 \%$, and a rise in the number of potential users by 5.5 percent. This was achieved as part of a monitoring scheme. The clothes have an electric circuit that causes a current of more than 3000 volts, which will protect women from harassment and allow them to flee from harassers while remaining safe [11]. As technology advances, we are witnessing everyday new technologies for our convenience. Recently, a smart belt was proposed; it is a compact device that functions similarly to a traditional belt. This belt contains an Arduino board, a screaming bell, and pressure sensors that will sound an alarm if the pressure exceeds the set limit [12]. Aeshs is the most recent and upgraded device with cutting-edge technology; it can locate the exact location of the suspected victim, as well as detect the immediate area in which the victim is abused or harassed. This will aid law enforcement officers in identifying the individual who assaulted or assaulted the women, and appropriate action will be taken. This device will now also have cast and live status [13].

\section{RESEARCH METHODOLOGY}

The main purpose of this research is to be used by women concerned individuals, be it caretakers or parents, to alert their parents as when required. By using this system, it can avoid any physical loss or kidnapping of a woman. This system is developed by using simple components (see figure 3.1). This system has an interface with Arduino. Open-source solutions were proposed to construct this system and others used in the proposed safety alert system can be easily integrated with the Arduino platform.

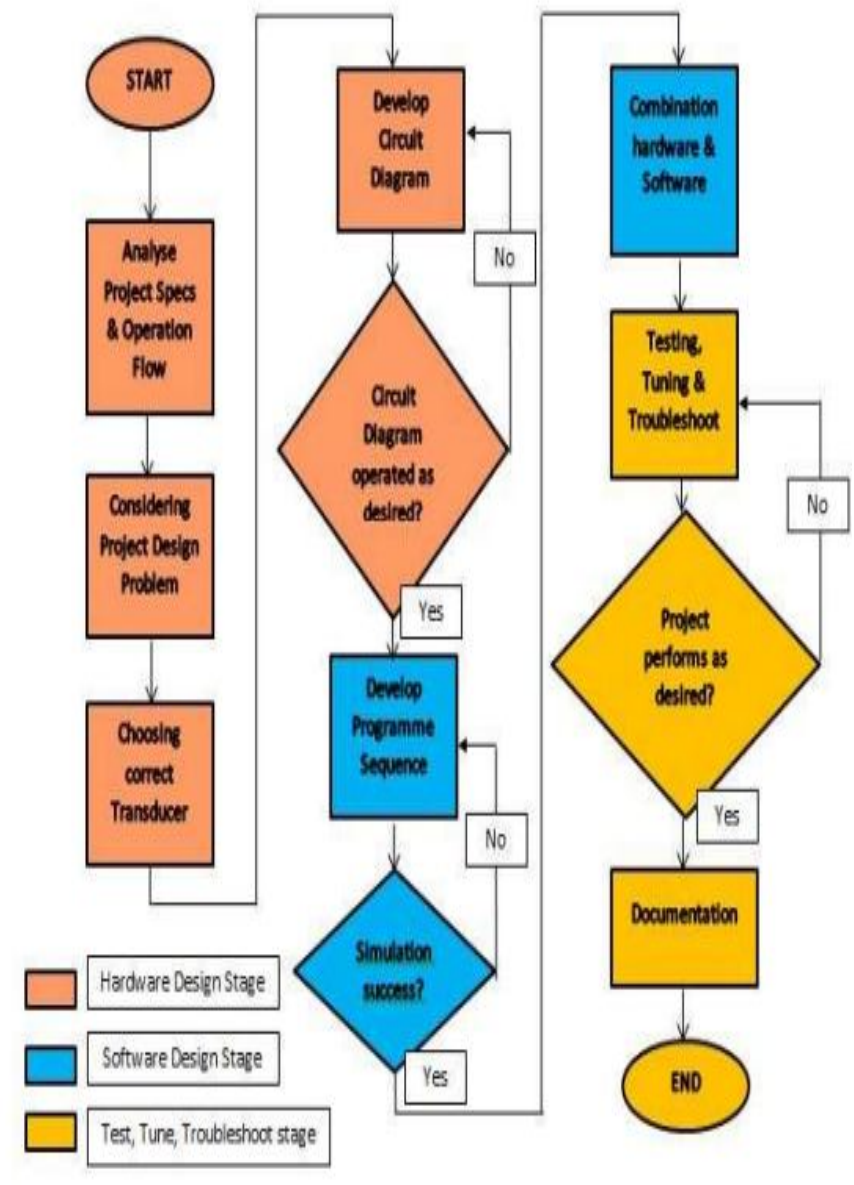

Figure 3.1: Flow of Design Stages of Study 
The design specification as shown in Figure 3.1 is determined based on the objective of the project. The project is divided into three stages. initialize with the hardware design stage, then the software design stage and the last step is testing, tuning and troubleshooting the project design. The hardware design stages act as tools to determine whether the transducer used is correct and compatible with the circuit diagram. In this stage, the transducer is chosen based on experimental characteristics such as accuracy, precision, factors affecting the measurement and the performance under forced conditions. The software design stage is designed according to the operation at the flow of the project. The software part itself is divided into two categories, which are the sequence programming and the interface programming. Both parts must be joint together and run simultaneously in order to produce the approved intention of the project. The testing, tuning, and troubleshooting stages are the keys to the design process. These stages occur after the combination of both the hardware and software components. Therefore, a slight error in design can be time-consuming and may result in retracing to previous stages for affirmation.

\subsection{CASE STUDY 1: How to TRaCK YOUR WOMEN}

\section{LOCATION WITH SMS}

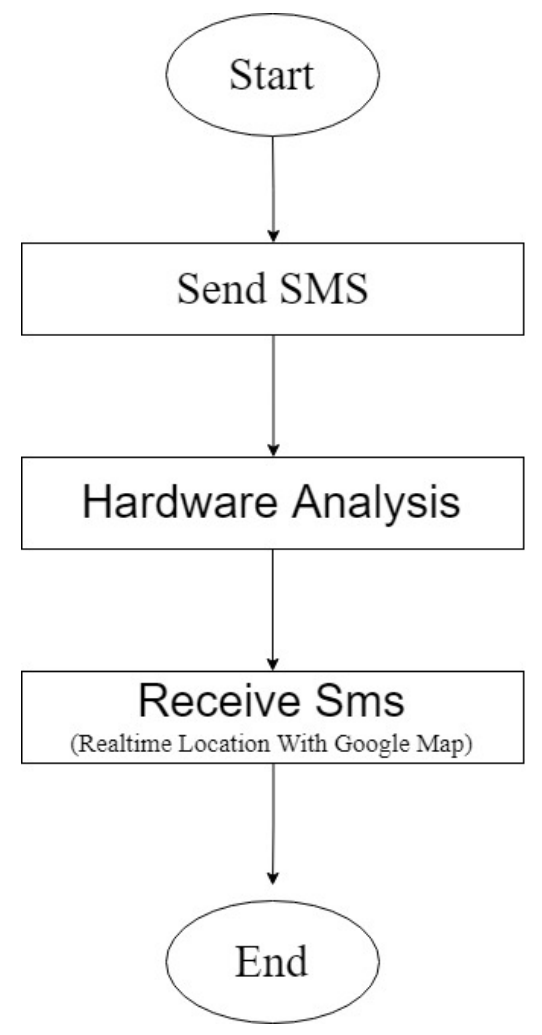

Figure 3.2: How to track your women location with SMS

\subsection{CASE STUDY 2: HOW TO TRACK YOUR} WOMEN LOCATION WITH THE INTERNET

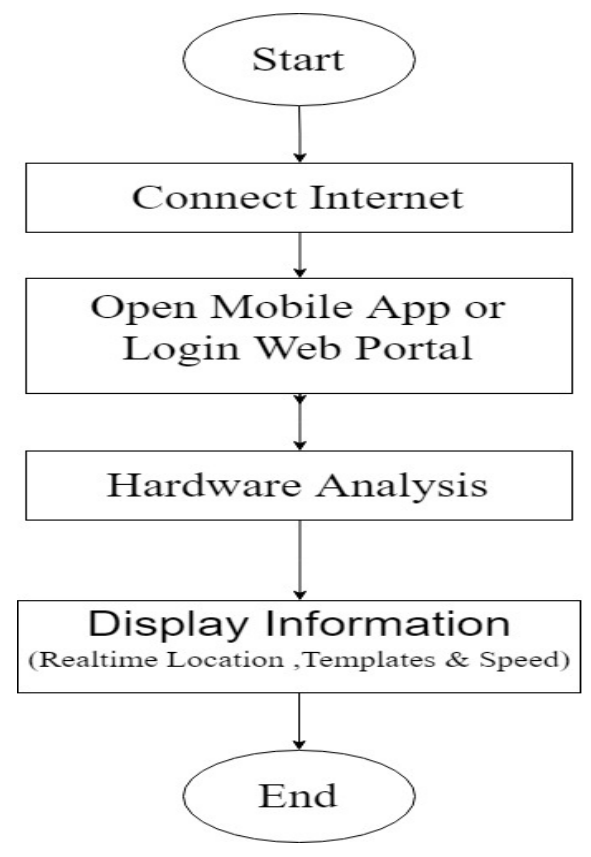

Figure 3.3: How to track your women location with Internet 3.3 CASE STUDY 3: WOMAN AND LADY CAN
PRESS THE BUTTON THEN SHOCK IS GENERATED
AT THE TIP OF THE SANDAL

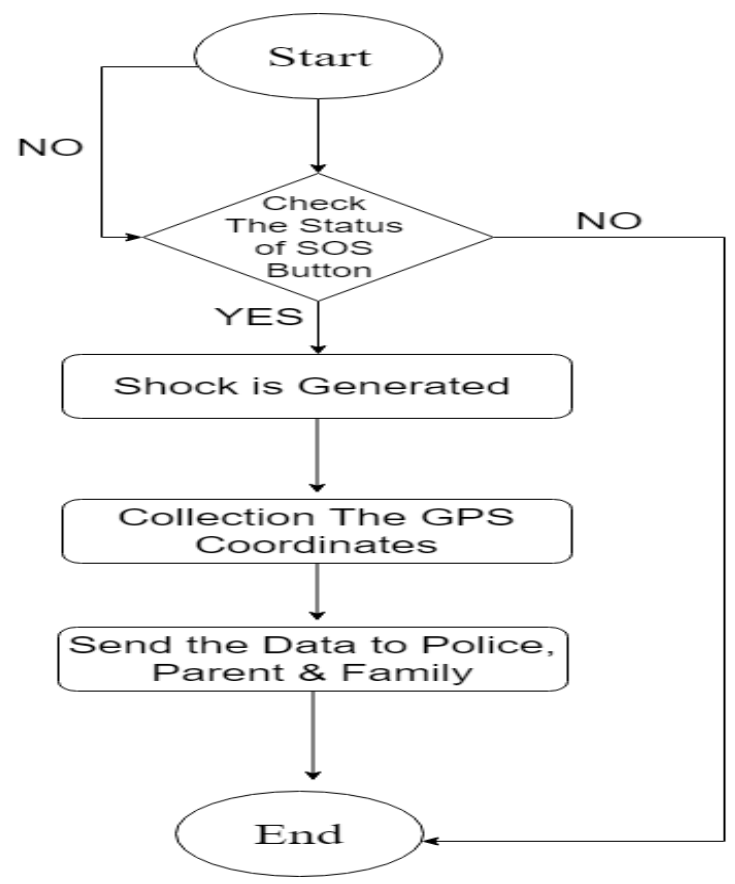

Figure3.4: Woman and Lady can press the button then Shock is generated at the tip of the sandal 


\subsection{CASE STUDY 4: PRESS SOS BUTTON SYSTEM SEND LOCATION WITH SMS \& INTERNET}

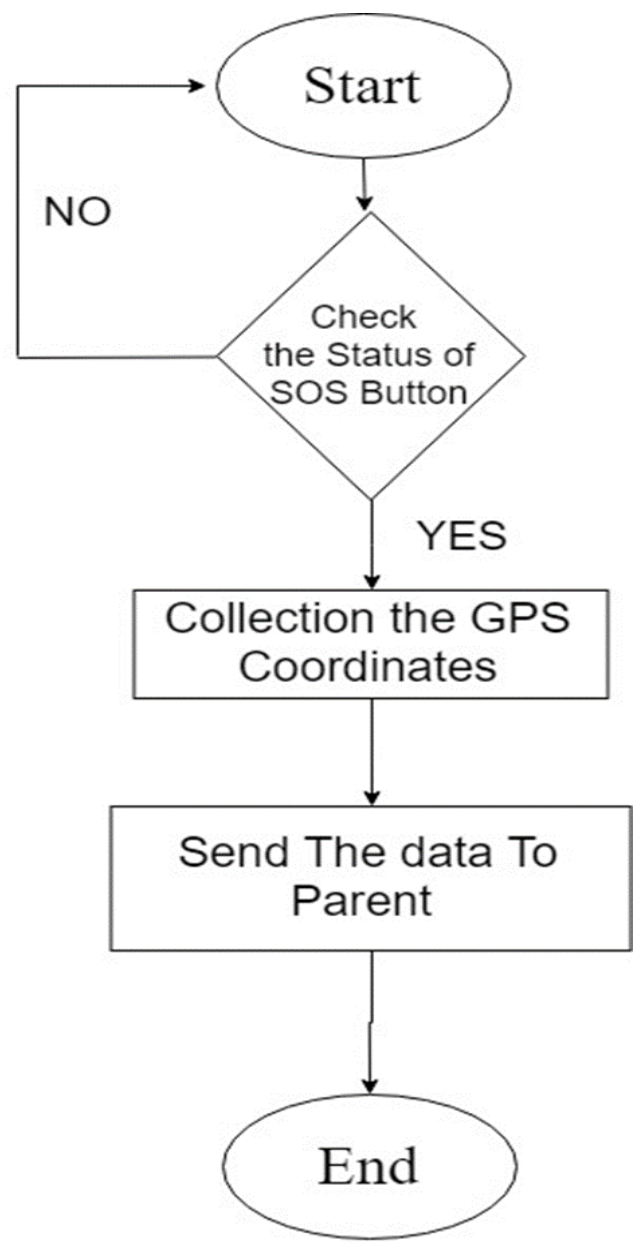

Figure 3.5: Press SOS button System send Location with SMS \& Internet

\section{IMPLEMENTATION \& EXPERIMENTS}

The research is divided into three stages. initializing with the hardware design stage, then the software design stage and the last step is testing, tuning and troubleshooting the project design.

\subsection{HARDWARE IMPLEMENTATION}

This system has an interface with ESP8266. Open-source solutions were proposed to construct this system and others used in the proposed safety alert system can be easily integrated with the Arduino platform.

\subsubsection{FLOW OF HARDWARE SECTION}

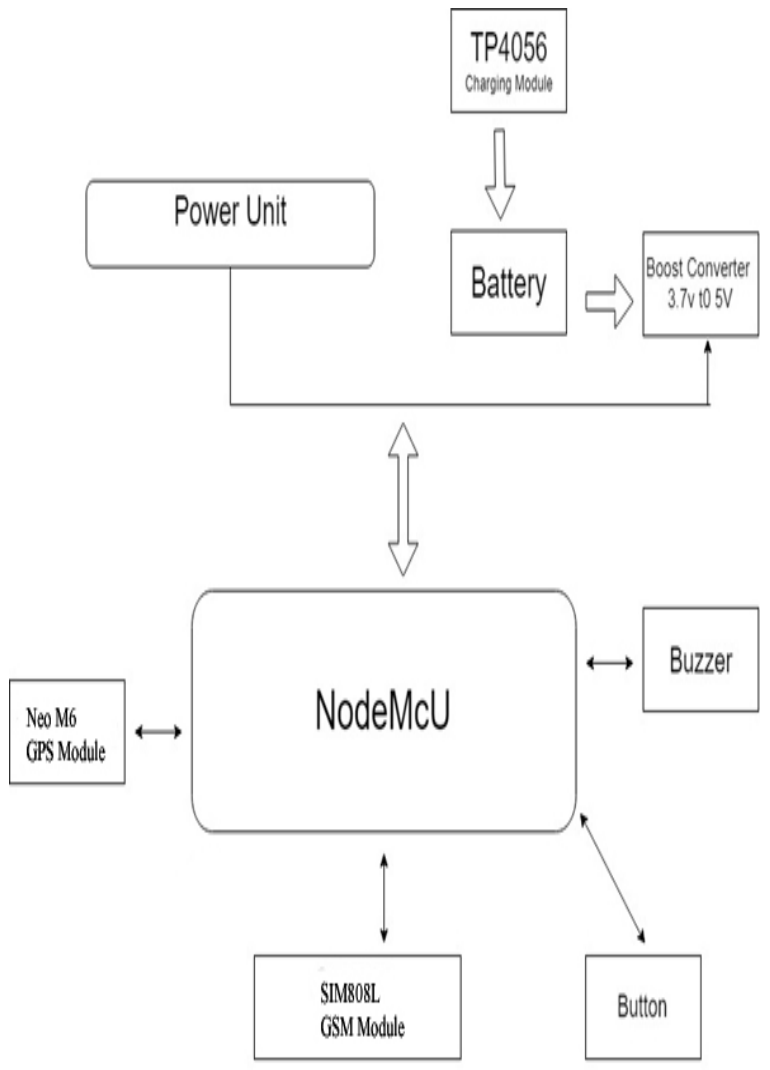

Figure 5.1: Hardware Selection Working Flow.

\subsubsection{ELECTRIC SHOCK GENERATOR}

This shock generator (see Figure 5.2) is an electric device which develops voltages approximately $1200 \mathrm{mv} \&$ current of 3 microamp. Electric Shock generator is fixed in the sandal. As soon as the push button is pressed the shock is generated in the sandal. Portable Device that will generate a shock. After that, location will be traced with GPS and sent over to relatives by GSM module. Call will also be placed

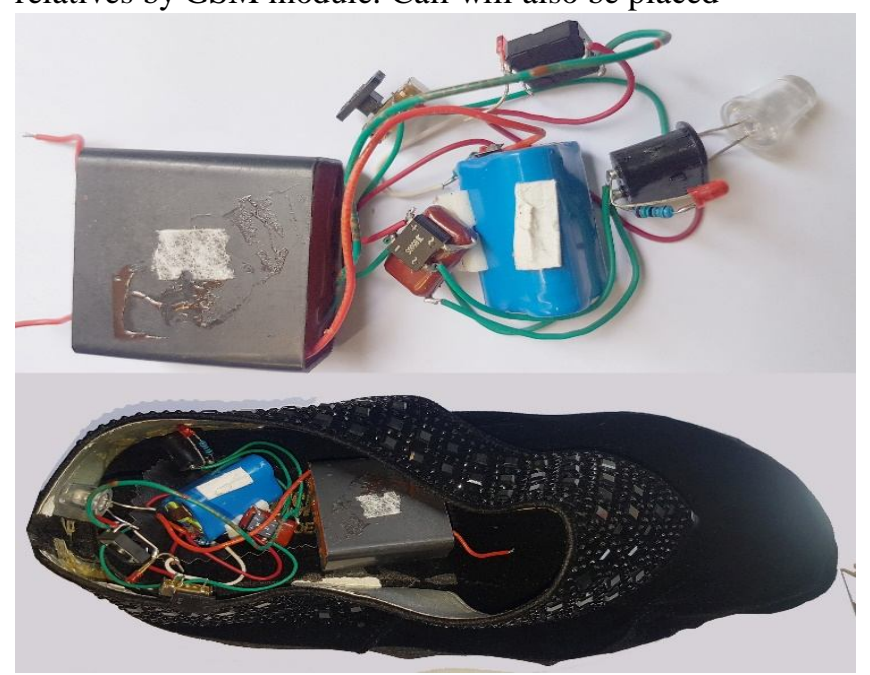

Figure 5.2: Electric Shock Generator with Sandal 


\subsubsection{TP4056 - (BATTERY CHARGER MODULE WITH PROTECTION)}

The small module (see figure 5.3) is best for charging a single cell higher LiPo cells or 3.7V $1 \mathrm{Ah}$. Because of the capacity of the TP4056 charger IC and DW01 battery protection, the 1A circuit is cut off when it finishes. When the battery's voltage drops, it automatically reduces the load and protects the battery.

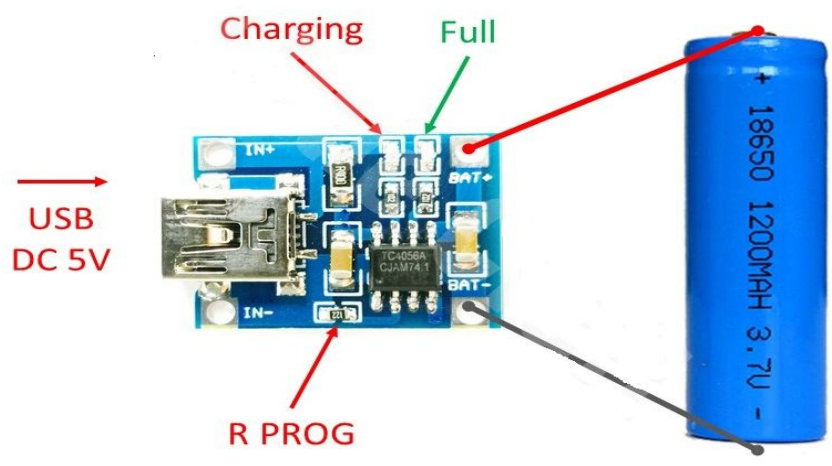

Figure 5.3:TP4056 Module

\subsubsection{RECHARGEABLE LI-ION BATTERY}

Robots are no longer limited to bulky, low powered, non-rechargeable batteries. The introduction of portable personal electronics has been a massive surge in battery research within the last decade. A Lithium polymer battery is used. Since the module is autonomous, the power supply should be portable. The capacity of the power supply is $7.4 \mathrm{~V}$ and it is rechargeable.

\subsubsection{PANIC BUTTON}

The panic button is a combination of a connector(see figure 5.4), LED, and two resistors that tend to pull up all these objects are made on the PCB. When the woman presses the button, its coordination has been sent to her relatives' parents.

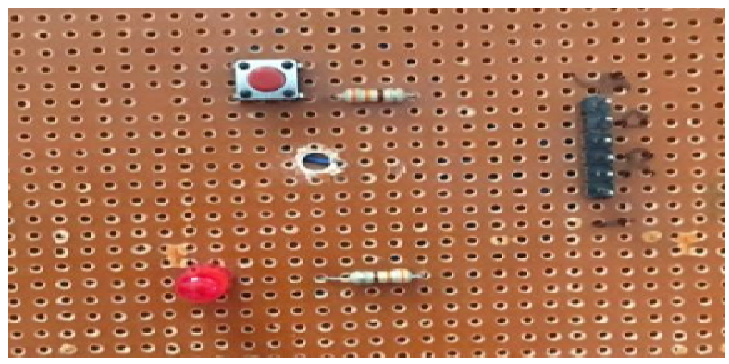

Figure 5.4: Panic Button Circuit

\subsubsection{BUZZER}

There are two pins on the Buzzer (see figure 5.5). The size of one pin differs from the size of the other. They are normally linked to the larger. The ground is attached to the smaller screw. The buzzer starts beeping when the microcontroller pin connected to the larger pin of the buzzer goes heavy.

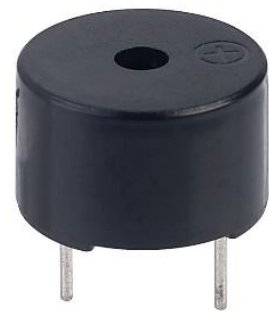

Figure 5.5: Buzzer

\subsection{SOFTWARE IMPLEMENTATION}

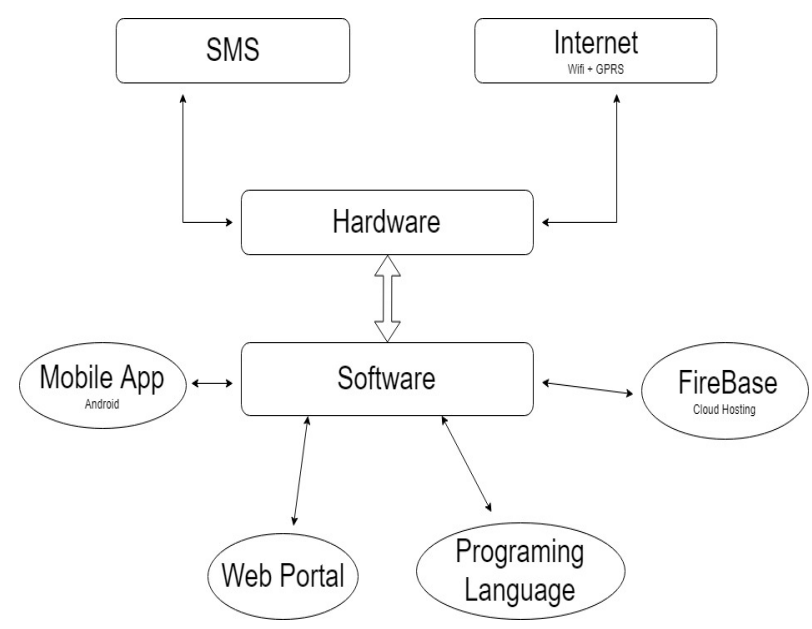

Figure 5.6: Software Design

\subsubsection{ARDUINO SOFTWARE)}

The Arduino Freeware Software (IDE) compiles the code and transfers it to the pursuit industry. It sustains Windows, Linux and Mac OS X. In-direct as comic Esp8266 panel through a comfortable combination of form worn with achieving real task (cipher) using ultimate transferred comic Esp8266.The Arduino community is opposed to peaceful shareware, and this prevents the publication of each cipher as a scene performed per person Arduino-Esp8266 and related to the board. The full cause code is Esp8266.

\subsubsection{ANDROID}

The Android is an operating system that has software integrated into mobile devices. On a larger scale, Android is an ecosystem created by Google and its affiliates, which allows Android developers to create, distribute and monetize applications for a variety of Android touchscreen devices. In 
addition, the touch screen is configured. Google has designed to develop the TV, has Android specifications and is exceptionally easy to use for users.

\subsubsection{JAVA}

Java is the programming language most used by Android developers. James Gosling of Sun Microsystems originally developed it in 1995, which is now acquired by Oracle Corporation. One of the main reasons for using Java for the development of Android is the quality of the platform independent of the language. Android works on many different hardware platforms. Therefore, the use of any language dependent on the platform will lead you to compile and optimize your native code for each of these different platforms to see all the real advantages.

\subsubsection{ANDROID SDK}

The set of comprehensive tools of development is presented in the Android development kit SDK. These tools of development include libraries, documentation, QEMU, and tutorials. The Android SDK has supported multiple platforms such as Linux, Window 7 and Mac OS X

\subsubsection{GOOGLE FIREBASE}

The Google Firebase Internet and mobile application that provide various platforms for the development Invented by Firebase, Inc. in 2011, then acquired by Google in 2014. We have used a handful of features provided by this platform in our project, which made our work for building the server side of our application a lot easier. The Firebase services that we use for building the server side of our mobile application are briefly discussed below:

- Authentication: We have used the authentication module of Firebase to manage our users and securely. Firebase Author offers multiple methods to authenticate, including email/password, third-party providers like Facebook or Google, or using your existing account system directly. We used the user's email and password for authentication and registration. We have built our interface, instead of taking advantage of their open source, fully customizable UI to make our UI design consistent and indifferent to other applications.

- Cloud Storage: Regardless of the quality of the network, the file has been downloaded as well as uploaded, with the help of Cloud Storage that has contained Firebase SDKs with Google security. For these reasons, we took advantage of this cloud storage for storing our users' profile pictures, which will help us to identify each user more accurately.

- Google Analytics: Another feature that comes with Firebase is the Analytics module, which contains the behavior as well as the attributes of the user that has been presented on the dashboard and defined in the form of a roadmap of the specific product. We got many insights such as the number of our monthly active users, percentages of user engagements on different activities of the application, region wise usage of our application and many more.

\subsubsection{THIRD PARTY LIBRARIES AND APIS}

The API of the Google Android MAP has added the maps that are based on the Google Maps information and data to integrate with the application. The API involuntarily handles way into the servers of Google Maps, map display, data downloading as well as giving a response to the map gesture.

\subsubsection{GOOGLE MAPS DIRECTION API}

The API of Google Map Directions has determined the directions integrated with the locations. To indicate the directions for multiple modes, including transit, transportation, driving, cycling, walking, etc.

\subsubsection{MQ TELEMETRY TRANSPORT (MQTT)}

MQTT is a TCP/IP-based published subscribe architecture that allows bidirectional communication between a device and an MQTT broker. MQTT is more suitable for constrained devices with limited resources than HTTP because it has only a minimal packet overhead with a very lightweight protocol. On the client side, its installation is very easy. The client-based server model is placed at the alternative of the publisher and the subscribers. In the pub/sub model, the publisher (who sends the message) is completely decoupled from the subscriber (who receives the message), which means that the clients do not know about the presence of further clients. Both the publisher and the subscribers have identified the other party, known as the broker. It handles and manages all the messages, filters and distributes them accordingly.

\section{RESULTS}

The shock generator circuit is located in the sandal, making this a compact device. This is depicted in the diagram below.

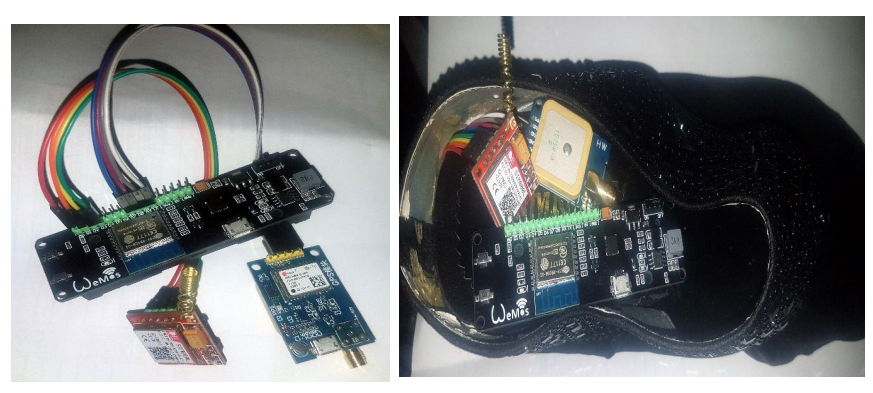

Figure 6.1: Smart Sandal Interface 
The rest of the circuit is plugged into a Smart Sandal. The woman will trigger the push-button switch if someone attempts to threaten her. A path is created and a discharge is produced at the tip of the sandal as soon as the button is pressed. The microcontroller will send a signal to the GPS and GSM via Esp8266 (Android + web application interface) as the discharge is induced. The GPS will send the woman's location (latitude and longitude) to GSM, and GSM will send a message requesting assistance. As you can see, it's as follows. If that number does not check the message, the machine will continue to call that number whenever he or she does not reject the call. This is the system's most significant benefit. During programming, we can also add the ' $n$ ' numbers. If the first number is not labelled, the call and message will be forwarded to the next, and so on. As a result, we will ensure women's protection.

\section{CONCLUSION \& FUTURE WORK}

The key emphasis of this paper was on the protection of women in public places. Smart Sandal is created by combining the various modules that make up the device. This device uses a GSM and GPS module to transmit an emergency alert to stored contacts, along with the latest longitude and latitude values, as well as an electric shock. When a circumstance of aggression is detected, the device can be triggered by pressing a button. By pressing the Button, the device would activate GSM and GPS modules, as well as Electric Shock and Buzzer, causing the attacker to lose his veering and allowing the public to contact the attacker. However, if the victim only wants to submit her location, she can do so by pressing the button, which will only activate the GSM and GPS modules while turning off the other two output devices. The project was created with the aim of leaving no stone unturned in providing a healthy atmosphere for women in all circumstances. Here are the hardware and software components of the security assistance device, as well as how it works. The prototype can send and receive messages, and the device's location can be monitored using the Google Maps API. The developed system illustrates how the technology can assist women in distress in calling for assistance and law enforcement officers in assisting them in real-time. When improved, the system will provide a cost-effective device that can also be run globally. In the future, the device's size can be reduced by interfacing a combined GSM and GPS module with the NODE MCU to serve the device's function. Additional functionality can be added, and the system's stability can be enhanced. This prototype has the potential to be released on the market as a stand-alone product, as its use would be extremely beneficial to women.

This paper has primarily concentrated on one of society's most concerning problems, namely, women's protection and safety in Pakistan. We demonstrated a prototype using various modules in this study. The system passed the testing and proved to be effective. Since the device is low-cost, it is extremely successful and efficient. There is, however, still room for change. It may be appropriate to launch a new version with additional modules while making no significant changes to the overall system as technology advances or new user requirements to enhance the functionality of a product update. This method could be applied in a variety of ways with further study and creativity. The framework can be implemented in a variety of wearable devices. This device can be used as a safety locator wristband with GSM and GPS modules, a safety belt, and other applications. We can also create an Android application that displays the position directly so that the longitude and latitude values can be calculated. We may also provide a small camera to catch the attacker's image and assist the victim. The sandals can also be rendered waterproof.

\section{ACKNOWLEDGEMENT}

Authors would like to thank anonymous reviewers and chief editor of IJATCSE for their valuable suggestions which enhance the quality of paper.

\section{REFERENCES}

1. G.Bharathi, L. Ramurthy, Children Tracking System Using Arm7 Microcontroller, conference paper in International Journal of Industrial Electronics and Electrical Engineering, Dec 2014, vol. 2.

2. Geetha Pratyusha Miriyala, Smart Intelligent Security System For Women, International Journal of Electronics and Communication Engineering \& Technology (IJECET), Volume 7, Issue 2, March-April 2016.

3. M. Muteeb, J. Muhammad, Y. Sarosh, M. A. Yousaf, and S. Shahid, "Mehfoozaurat: Transforming smart phones into women safety devices against harassment," Eighth International Conference on Information and Communication Technologies and Development, ACM, New York, USA, 2016.

4. Ghaith Bader Al-Suwaidi, Mohamed Jamal Zemerly, "Locating friends and family using mobile phones with global positioning system (GPS)," IEEE/ACS International Conference on Computer Systems and Applications, 2009.

5. Chandra, A., Jain, S., Qadeer, M.A., "GPS Locator: An Application for Location Tracking and Sharing Using GPS for Java Enabled Handhelds," 2011 International Conference on Computational Intelligence and Communication Networks (CICN), pp.406-410, 79 Oct. 2011

6. Almomani, I.M., Alkhalil, N.Y., Ahmad, E.M., Jodeh, R.M., "Ubiquitous GPS vehicle tracking and 
management system," 2011 IEEE Jordan Conference on Applied Electrical Engineering and Computing Technologies (AEECT), pp.1-6, 6-8 Dec. 2011.

7. M.Navya, et.al , "Android based children tracking system using voice recognition”, International journal of Computer science and information technology, Vol 4 (1): pages 229-235, Jan 2015.

8. Huiyu Zhou, Huosheng $\mathrm{Hu}$, "Reducing drifts in the inertial measurements of wrist and elbow positions".IEEE Trans vol.59,no.3 (2010).

9. Liu, Kun, Liu, Tao, Shibata, K., Inoue, Y., Zheng, Rencheng, 2008. "Novel approach for lower limb segment orientation in gait analysis using triaxial accelerometers". IEEE/ASME International Conference on Advanced Intelligent Mechatronics (2008), 488-492.

10. S. Shafaat, UAE launches smart school buses to improve student's safety system to offer parents direct access to bus status". 1 (2). Available Online: http://www.emaratalyoum.com/local-section/education/ 2013-04-22-1.568158

11. lexandrous Plantelopoulous and Nikolaos.G.Bourbakis, "A Survey on Wearable sensor based system for health monitoring and prognosis," IEEE Transaction on system, Man and Cybernetics, Vol.40, No.1, January 2010.

12. B.Chougula, "Smart girls security system," International Journal of Application or Innovation in Engineering \& Management, Volume 3, Issue 4, April 2014. 